\title{
A Computational Basis for Conic Arcs and Boolean Operations on Conic Polygons ${ }^{\star}$
}

\author{
Eric Berberich, Arno Eigenwillig, Michael Hemmer, Susan Hert, \\ Kurt Mehlhorn, and Elmar Schömer \\ Max-Planck-Institut für Informatik \\ Stuhlsatzenhausweg 85, 66123 Saarbrücken, Germany \\ \{eric, arno, hemmer, hert, mehlhorn, schoemer\} @mpi-sb.mpg.de
}

\begin{abstract}
We give an exact geometry kernel for conic arcs, algorithms for exact computation with low-degree algebraic numbers, and an algorithm for computing the arrangement of conic arcs that immediately leads to a realization of regularized boolean operations on conic polygons. A conic polygon, or polygon for short, is anything that can be obtained from linear or conic halfspaces (= the set of points where a linear or quadratic function is non-negative) by regularized boolean operations. The algorithm and its implementation are complete (they can handle all cases), exact (they give the mathematically correct result), and efficient (they can handle inputs with several hundred primitives).
\end{abstract}

\section{Introduction}

We give an exact geometry kernel for conic arcs, algorithms for exact computation with low-degree algebraic numbers, and a sweep-line algorithm for computing arrangements of curved arcs that immediately leads to a realization of regularized boolean operations on conic polygons. A conic polygon, or polygon for short, is anything that can be obtained from linear or conic halfspaces (= the set of points where a linear or quadratic function is non-negative) by regularized boolean operations (Figure 1). A regularized boolean operation is a standard boolean operation (union, intersection, complement) followed by regularization. Regularization replaces a set by the closure of its interior and eliminates dangling low-dimensional features.

Our algorithm and implementation are complete and exact. They are complete in the sense that they can handle all inputs including arbitrary degeneracies. They are exact in that they always deliver the mathematically correct result. Complete and exact implementations for the linear case are available, e.g., in the generalized polygon class [21, Section 10.8] of LEDA and in the planar map class [15] of CGAL. However, existing implementations for conic polygons are either incomplete or inexact, except for the very recent work by Wein [25].

\footnotetext{
* Partially supported by the IST Programme of the EU as a Shared-cost RTD (FET Open) Project under Contract No IST-2000-26473 (ECG - Effective Computational Geometry for Curves and Surfaces).
} 

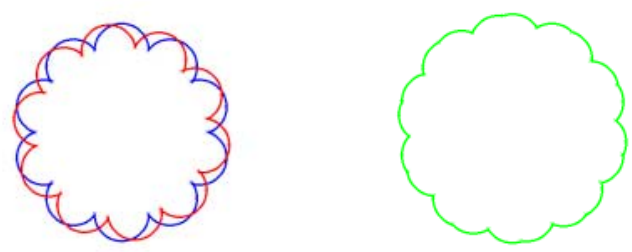

Fig. 1. We compute the union of two curved polygons (left panel). The first input polygon is created from a regular 10-gon by replacing each straight edge with a half-circle with that edge as diameter. The second polygon is created from the first by rotating it around the origin by $\alpha=\pi / 20$ radians. On the right is the union of these two polygons. We can compute the correct union of such rotated $n$-gons for any $n$ and any $\alpha$. For $n=1000$ and $\alpha=\pi / 2000$, the time required to compute the correct union with 2702 edges is less than 20 minutes on an $846 \mathrm{MHz}$ Pentium III processor

There are three main parts to our work: (1) a sweep-line algorithm for computing arrangements of curved arcs, (2) predicates and functions for conic arcs, and (3) algorithms for the exact computation with low-degree algebraic numbers. For part (1), the sweep-line algorithm extends the Bentley-Ottmann sweep-line algorithm for segments [1]. The handling of many curves passing through the same point is considerably more involved than in the straight-line case. For (2), we give algorithms for basic predicates and functions on conics and use them to realize the functionality required in the sweep algorithm. For (3), we have integrated the representation of algebraic numbers as roots of polynomials and the representation as explicit expressions involving square roots.

Our implementation consists of a basic layer providing polynomials, roots of polynomials, low-degree algebraic numbers, conics, and predicates and functions on conics and conic arcs and an algorithmic layer that provides arrangements of conics, the sweep-line algorithm for curves, and boolean operations on conic polygons and on polygons with circular and straight-line arcs. We have tested our implementations on inputs of various sizes and with various degeneracies; see for example Figure 2. We provide evidence of the efficiency of the approach presented here by comparing the results to the implementations in LEDA for polygons with line-segment edges. Our implementation can handle scenes with several hundred conic segments.

The rest of the paper is organized as follows. We first summarize related work (Section 2) and then review the Bentley-Ottmann sweep-line algorithm. We extend it to curved arcs and derive the required set of predicates and functions (Section 3). In Section 4 we discuss conics, functions and predicates on conics, and computation with low-degree algebraic numbers. In Section 5 we give more details of our implementations and provide the results of our experiments. Section 6 offers some conclusions. 


\section{Related Work}

The work of three communities is relevant for our work: computational geometry, solid modeling, and computer algebra. The solid modeling community has always dealt with curved objects, and CAD systems dealing with curved objects in two and three dimensions have been available since the 60's. None of these systems is complete or exact, not even for straight-line objects. The question of complete and exact implementations has been addressed only recently. MAPC [20] provides a set of classes for manipulating algebraically defined points and curves in the plane, which includes an implementation of the naïve $O\left(n^{2}\right)$ algorithm for computing an arrangement of $n$ curves in the plane. The algorithms are not complete; they handle some but not all degeneracies. Also the use of Sturm sequences to handle degenerate cases, such as tangential curves or degenerate segments, results in unnecessarily slow computations in these cases. ESOLID [19] performs accurate boundary evaluation of low-degree curved solids. It is explicitly stated that degeneracies are not treated.

Surprisingly little work in computational geometry deals with curved objects; some examples are [24, 12, 11]. The sweep-line algorithm of Bentley-Ottmann [1] is known to work for $x$-monotone curves, at least in the absence of degeneracies. Degeneracies have been discussed for straight-line segments only. Several papers [3, 4, 6] have looked into the question of using restricted predicates to report or compute segment intersections, the rationale being that lower-degree predicates are simpler to evaluate. All papers have to exclude at least some degenerate cases.

The exact and efficient implementation of the predicates required in our algorithms is non-trivial, since they involve algebraic numbers. One of the predicates used in our algorithms, the lexicographical comparison of vertices in an arrangement of circles and lines, has been considered by Devillers et al. [10]. A very efficient realization is given. The more complex predicates also needed in our algorithms are not discussed in their work and it is not clear whether the technique generalizes.

CGAL's planar map class also supports the computation of arrangements of circular arcs and line segments. Very recently, the implementation was extended to conic arcs by Wein [25]. The implementation is in some respects similar to ours. However, the sweep method is not yet available and the the computation of boolean operations on polygons is implemented only indirectly.

The papers $[13,17]$ show how to compute arrangements of quadrics in threespace. The algorithms are complete, but (as of now) can handle only a small number of quadrics. Root isolation of univariate polynomials, resultant computation, and exact treatment of algebraic numbers are well studied problems in computer algebra. We use the standard techniques.

\section{Conic Polygons and the Sweep-Line Algorithm}

The Bentley-Ottmann Sweep-line Algorithm: The Bentley-Ottmann sweep-line algorithm for computing an arrangement of segments in the plane [1] was origi- 
nally formulated for sets of segments, no three of which pass through a common point and no two of which overlap each other. A vertical line is swept across the plane and the ordered sequence of intersections between the sweep line and the segments is maintained (= Y-structure). The status of the sweep line changes when a segment starts, when a segment ends, and when two segments cross. Bentley and Ottmann observed that the algorithm can actually handle any set of $x$-monotone curves. Of course, when two such curves meet they may either cross or touch, which requires a minor modification to the algorithm. The events are maintained in a priority queue, referred to as the X-structure.

It was later observed, see for example [9, 21], that in the case of straightline segments the algorithm can also handle arbitrary degeneracies. A number of small extensions are required. For example, when the algorithm sweeps across a point in which several segments meet, the $y$-order of the segments meeting at this point is reversed.

We next argue that the algorithm can also handle degenerate situations in the case of curves. The main problem is sweeping across a point where many curves meet and we restrict our attention to this problem here.

Consider a point $p$ and assume that $\operatorname{arcs} C_{1}$ to $C_{k}$ pass through $p$. We assume that the curves are numbered according to their $y$-order just left of $p$. Let $s_{i}$ be the multiplicity of intersection of the curves $C_{i}$ and $C_{i+1}$ at $p$; see [2, Chapters I and IV] for a formal definition. Intuitively, the multiplicity is one if the curves meet at $p$ and have different slopes, the multiplicity is two if the curves have identical tangent but different radii of curvature, the multiplicity is three if the curves have same tangent and identical radii of curvature but different ... . Two curves meeting at $p$ cross at $p$ if the multiplicity of the intersection is odd, and they touch, but do not cross, if the multiplicity is even. The multiplicity of intersection between $C_{i}$ and $C_{j}$ for $i<j$ is $\min \left\{s_{i}, \ldots, s_{j-1}\right\}$ because the multiplicity of intersection is the number of identical initial coefficients in the local Taylor series expansion. For distinct conics, the multiplicity of intersection at any point is at most 4 . For example, the multiplicity of intersection at the origin between $y(1-x)=x^{2}$ and $y=x^{2}+y^{2}$ is three.

The following algorithm determines the $y$-order of our curves $C_{1}$ to $C_{k}$ just to the right of $p$ in time $O(k)$. Make four passes over the sequence of curves passing through $p$. In the $j$-th pass, $4 \geq j \geq 1$, form maximal subsequences of curves, where two curves belong to the same subsequence if they are not separated by a multiplicity less than $j$, and reverse the order of each subsequence.

Lemma 1. The algorithm above correctly computes the y-order of the segment passing through a common point $p$ immediately to the right of $p$ from the order immediately to the left of $p$.

Proof. Consider two arbitrary curves $C_{h}$ and $C_{i}$ with $h<i$. Their $y$-order right of $p$ differs from their $y$-order left of $p$ iff $s=\min \left\{s_{h}, \ldots, s_{i-1}\right\}$ is odd. Next observe that $s$ is also exactly the number of times $C_{h}$ and $C_{i}$ belong to the same subsequence, i.e., the number of times their order is reversed. We conclude the order of $C_{i}$ and $C_{h}$ is reversed iff $C_{i}$ and $C_{h}$ cross at $p$. 
Of course, the algorithm just outlined will also work if the maximal multiplicity $M$ of intersection is arbitrary. However, its running time will be $M$ times $k$, the number of curves passing through the point. Lutz Kettner (personal communication) has shown that the permutation can always be computed in time linear in the length of the subsequence.

Conic Polygons: Regularized boolean operations on straight-line or conic polygons can be built on top of the sweep-line algorithm for segment intersection, see [21, Section 10.8]. The corresponding data structure in LEDA is called generalized polygons. We reused it with only one small change. When curved edges are used, a polygonal chain with only two edges is possible. We also made the implementation more general by parameterizing it with the type of the polygon used to represent the boundaries (Section 5).

Required Predicates and Functions: We are now ready to summarize the predicates and functions that must be defined for the points and segments used in the sweep-line algorithm and in the computation of generalized polygons.

compare_xy $(\boldsymbol{p}, \boldsymbol{q})$ - compares points $p$ and $q$ lexicographically. This predicate is used to maintain the order of the events in the X-structure.

seg.y_order $(\boldsymbol{p})$ - determines if a point $p$ in the $x$-range of segment seg is vertically above, below, or on seg. This predicate is used to insert starting segments into the Y-structure.

seg.compare_right_of_common_point(seg2, p) - compares seg and seg2 to determine their $y$-ordering just right of their common point $p$. This predicate is also used to insert starting segments into the Y-structure. The order of segments starting at the same point is determined by this predicate.

seg.common_point_multiplicity(seg2, p) - returns the multiplicity of intersection of seg and seg2 at their common point $p$. This predicate is used to handle curves passing through a common point as described above.

seg.has_on $(\boldsymbol{p})$ - determines if $p$ lies on seg. This predicate is not needed in the sweep line algorithm, but in the algorithm for boolean operations. It is used to determine the containment of one boundary inside another.

intersect(seg1, seg2, result) - determines if seg1 and seg2 intersect or not and, if so, inserts their intersection points in lexicographical order into result

\section{Conics and Computations with Conics}

We discuss predicates and constructions on conics and their algorithmic realization in this section.

Every conic is defined as the zero-set in $\mathbb{R}^{2}$ of a quadratic implicit equation $P$ in the variables $(x, y)$ i.e.,

$$
\alpha_{1} x^{2}+\alpha_{2} y^{2}+2 \alpha_{3} x y+2 \alpha_{4} x+2 \alpha_{5} y+\alpha_{6}=0
$$

with $\neg\left(\alpha_{1}=\alpha_{2}=\alpha_{3}=0\right)$. We restrict attention to the non-degenerate conics in this paper as lines bring nothing new algorithmically. For every value $x$ there are 
at most two values of $y$ satisfying the equation. We can obtain an $x$-monotone parameterization by rewriting the equation as a quadratic equation for $y$ and solving for $y$. We obtain $Q=a(x) y^{2}+b(x) y+c(x)=0$. Solving for $y$ yields

$$
y= \begin{cases}\frac{-b(x) \pm \sqrt{b(x)^{2}-4 a(x) c(x)}}{2 a(x)} & \text { if } \alpha_{2} \neq 0 \\ \frac{-c(x)}{b(x)} & \text { if } \alpha_{2}=0 \text { and } b(x) \neq 0\end{cases}
$$

Degenerate conics are easily recognized [18].

A conic arc is an $x$-monotone curve that corresponds to one choice of sign in the equation above. A conic decomposes into either one or two conic arcs. In the latter case we refer to the two arcs as the lower and the upper arc. For a conic $C$ we use $C_{0}$ and (if it exists) $C_{1}$ to denote the arcs of $C$. We view the arcs as functions of $x$, i.e., $C_{1}(x)$ is the $y$-value of the upper $\operatorname{arc}$ of $C$ at $x$.

Intersection of Two Conics: Consider two conics

$$
Q_{1}=a_{1} y^{2}+b_{1} y+c_{1}=0 \quad Q_{2}=a_{2} y^{2}+b_{2} y+c_{2}=0 .
$$

Then there is a polynomial $R$ in $x$ of degree at most four such that the $x$ coordinates of the intersections of $Q_{1}$ and $Q_{2}$ are roots of $R$.

$$
R=a_{2} b_{1}^{2} c_{2}+a_{2}^{2} c_{1}^{2}-a_{2} c_{1} b_{2} b_{1}-2 a_{2} a_{1} c_{1} c_{2}-b_{2} a_{1} b_{1} c_{2}+c_{2}^{2} a_{1}^{2}+b_{2}^{2} a_{1} c_{1}=0
$$

is called the resultant of $Q_{1}$ and $Q_{2}$; see [8] for a discussion of resultants. A root $x$ of $R$ does not necessarily correspond to an intersection of $Q_{1}$ and $Q_{2}$ in $\mathbb{R}^{2}$; the corresponding $y$-values may have non-zero imaginary parts.

Low-Degree Algebraic Numbers: The $x$-coordinates (and similarly the $y$ coordinates) of intersections points are roots of polynomials of degree at most four. We call an algebraic number a one-root-number if it is of the form $\alpha+\beta \sqrt{\gamma}$ with $\alpha, \beta, \gamma \in \mathbb{Q}$. The following well-known lemma is useful.

Lemma 2. A degree-four polynomial $p$ either has four simple roots or all roots of $p$ are one-root-numbers. The two cases are easily distinguished and the oneroot-numbers can be determined in the latter case.

Proof. (Sketch) Follows from a case distinction on the degree of $p / \operatorname{gcd}\left(p, p^{\prime}\right)$.

We represent algebraic numbers $x$ in one of two ways. Either as one-rootnumbers or as triples $(P, l, r)$ where $P$ is a polynomial with only simple roots, $l$ and $r$ are rational numbers, $P$ has exactly one real root in the open interval $(l, r)$ and $P(l) \neq 0 \neq P(r)$. Such an interval is called an isolating interval for the root. In our implementation, $l$ and $r$ have the additional property that their denominators are powers of two. We determine isolating intervals by means of Uspensky's algorithm [7, 23]. Isolating intervals are easily refined by considering the point $m=(l+r) / 2$. If $P(m)=0$, we have a one-root-number for $x$. Otherwise, we replace the isolating interval by either $(l, m)$ or $(m, r)$ depending on the sign of $P(m)$. 
One-root-numbers are represented as objects of the number type leda-real, cf. [5] or [21, Section 4.4]. Integers are leda-reals, and if $x$ and $y$ are leda-reals, so are $x \pm y, x * y, x / y$, and $\sqrt[k]{x}$ for arbitrary integer $k$. Leda-reals have exact comparison operators $\leq,<$ and $=$. In particular, if $x$ is a leda-real and $P$ is a polynomial with integer coefficients, we can determine the sign of $P(x)$.

We next describe how to compare two algebraic numbers $x$ and $y$. If both of them are given as leda-real, we use the comparison operator of leda-real. If $x=(P, l, r)$ and $y$ is a leda-real, we proceed as follows: If $y \leq l$ or $y \geq r$, the outcome of the comparison is clear. So assume $l<y<r$. If $P(y)=0, x=y$. So assume that $P(y) \neq 0$. Then(!!!) $x \neq y$. We now refine the isolating interval for $x$ as described in the preceding paragraph until $y \notin(l, r)$.

In order to compare two algebraic numbers $x=\left(P, l_{x}, r_{x}\right)$ and $y=\left(Q, l_{y}, r_{y}\right)$ we have to work slightly harder. If the isolating intervals are disjoint, we are done. Otherwise, let $I=(l, r)$ be the intersection of the isolating intervals. We have $x=y$ iff $P$ and $Q$ have a common root in $I$. We first refine the isolating intervals of $x$ and $y$ using the endpoints of $I$. Then it is either the case that both intervals are $I$ or the intervals are disjoint. If they are disjoint, then we are done. Otherwise, we know that $P$ and $Q$ both have exactly one simple root in $I$. These roots are equal if $g=\operatorname{gcd}(P, Q)$ has a root $z$ in $I$, in which case $z$ must be a simple root. Thus the degree of $g$ can be used to decide quickly about equality or inequality in certain cases. For example, if $\operatorname{deg}(g)=0$, we know that $x$ and $y$ are not equal, and similarly, if $\operatorname{deg}(g)=4$, we know they are equal. Otherwise we use the fact that $g$ has only simple roots. So $x=y=z$ iff $\operatorname{sign}(g(l)) \neq \operatorname{sign}(g(r))$. Furthermore, depending on the degree of $g$, a rational or one-root representation for $x$ and $y$ may be obtained if they are zeros of $g$, $P / g$, or $Q / g$ and if the respective degree is $\leq 2$.

The critical values of a conic are the roots of $h(x)$ if $\alpha_{2} \neq 0$ and is the root of $b(x)$ if $\alpha_{2}=0$. At the critical values the conic either has a vertical tangent or a pole. Critical values are one-root-numbers.

Intersection of Two Conics, Continued: Let $R$ be the resultant of conics $C$ and $D$ and let $x$ be a root of $R$. Do $\operatorname{arcs} C_{i}$ and $D_{j}$ intersect at $x$ ?

If $x$ is given as a leda-real, we simply compare $C_{i}(x)$ and $D_{j}(x)$ using ledareals. Otherwise, by Lemma $2, x=(R, l, r)$ is a simple root of $R$ and hence $\operatorname{arcs} C_{i}$ and $D_{j}$ cross at $x$ if they intersect at all. Let $(R, l, r)$ be the representation of $x$. We refine the representation of $x$ until the isolating interval contains no critical value of either $C$ or $D$. Then $C_{i}$ and $D_{j}$ are defined on the entire interval $[l, r]$ and they intersect at most once in $[l, r]$. Thus $C_{i}$ and $D_{j}$ intersect at $x$ iff the signs of $C_{i}(r)-D_{j}(r)$ and $C_{i}(l)-D_{j}(l)$ differ. We compute the signs using leda-reals.

Conic Points, Conic Segments, and Comparisons: We specify conic points by an $x$-coordinate $\left(=\right.$ an algebraic number) and a conic arc $C_{i}$. The point has coordinates $\left(x, C_{i}(x)\right)$. If $x$ is a leda-real, we can compute the $y$-coordinate as a leda-real. A conic segment is the part of a conic arc between two conic points. 
The $x$-compare of two conic points is tantamount to the comparison of two algebraic numbers, which was discussed above. We turn to the $x y$-compare. Let $\left(x_{1}, C_{i}\right)$ and $\left(x_{2}, D_{j}\right)$ be two conic points. If $x_{1} \neq x_{2}$, we are done. If $x_{1}=x_{2}$ and we know a one-root-number for $x_{1}$, we simply compare $C_{i}\left(x_{1}\right)$ and $D_{j}\left(x_{2}\right)$. So assume otherwise. We compute the resultant $R$ of $C$ and $D$ and compare $x_{1}$ to the roots of $R$. Three cases arise: $x_{1}$ is either not a root of $R$, a multiple root of $R$ (yielding a one-root-number for it), or a simple root of $R$. In the latter two cases, we proceed as described in the paragraph on intersection of two conics. In the first case, we refine the isolating interval of $x_{1}$ until is contains no critical values of $C$ and $D$ and then use the fact that the $y$-order of the two arcs at $x_{1}$ is the same as the $y$-order at the right end of the isolating interval of $x_{1}$. The strategy just described also resolves the has_on predicate as well as y_order.

Multiplicity of Intersection: Let $p=(x, y)$ be an intersection point of $\operatorname{arcs} C_{i}$ and $D_{j}$. Then $x$ is a zero of the resultant of $C$ and $D$ with multiplicity $m>0$. If $m=1$, the multiplicity of the intersection is 1 . So assume that $m>1$. Then we know a one-root-number for $x$. We have to deal with two difficulties. The first difficulty is that there might be intersections with non-real $y$ values, but this cannot happen since the $y$ values would come in conjugate pairs. Thus the conic would have three points on the complex line through $x$ parallel to the $y$ axis and hence the conic would be degenerate.

The second difficulty arises when both conics have two arcs and hence hence there might be intersections between $C_{1-i}$ and $D_{1-j}$ at $x$. Let $d$ be the (currently unknown) multiplicity of the intersection at $p$ and let $e$ be the multiplicity of the intersection of $C_{1-i}$ with $D_{1-j}$ at $x$. Then $d+e=m$. We test $e \geq 1$ by testing the equality $C_{1-i}(x)=D_{1-j}(x)$ using leda-reals. If $e=0$, we are done. Otherwise, let $q$ be the corresponding intersection point. We next test $d \geq 2$, by checking whether the normal vectors $\left(C_{x}(p), C_{y}(p)\right)$ and $\left(D_{x}(p), D_{y}(p)\right)$ are parallel. Here $C_{x}$ and $C_{y}$ are the partial derivatives of $C$. The check is again a computation using leda-reals since $p=\left(x, C_{i}(x)\right)$. If $d<2$ then $d=1$ and we are done. So assume $d \geq 2$. If $m=3$ then $d=2$ and we are done. Assume otherwise, i.e., $m=4$. We check whether $e \geq 2$. If $e=1$ then $d=m-1=3$. If $e \geq 2$ then $d=e=2$.

Order Immediately to the Right of a Common Point: Consider $\operatorname{arcs} C_{i}$ and $D_{j}$ containing $p=(x, y)$ and extending to the right. Assume that we know a ledareal $u$ such that $x<u$, arcs $C_{i}$ and $D_{j}$ are defined on $[x, u]$ and do not intersect in the interval $(x, u]$. Then the $y$-order just right of $x$ is the same as the $y$-order at $u$; the latter can be computed using leda-reals. How can we obtain $u$ ? If $x$ is a simple root of the resultant of $C$ and $D$, we refine the isolating interval of $x$ until it contains no critical values of $C$ and $D$ and take $u$ as the right endpoint of the isolating interval. If $x$ is a multiple root of the resultant, we have oneroot-numbers for all roots and we simply take a rational point to the right of $x$ and within the $x$-range of the two arcs. This can be, for example, the midpoint of $x$ and the next larger root of the resultant if there is one. 


\section{$5 \quad$ Implementation and Empirical Results}

We describe our implementations and report about experiments. All our implementations are in $\mathrm{C}++$. They use components of LEDA, CGAL, and the standard template library.

Algebraic Numbers and Conics: We have implemented four classes: alg_number, conic, arrangement2, and X_mono_conic_segment. The first realizes algebraic numbers as discussed in Section 4. The main ingredients are polynomials, gcd of polynomials, resultant computations, Uspensky's algorithm for root isolation, and leda-reals. The main functionality is exact comparison between algebraic numbers. The class conic realizes conics and the predicates and constructions discussed in Section 4. The class arrangement2 uses the functionality of the two other classes to construct the arrangement of two conics. The class X_mono_conic_segment realizes an implementation of a segment type as required by the sweep-line algorithm and generalized polygon class.

The Naive Algorithm for Computing Arrangements of Conics: This algorithm takes a set of conics and computes the subdivision of the plane defined by them. It first computes the arrangement of any pair of conics and then merges the pairwise arrangements into a single arrangement. This implementation provides a testbed for classes alg_number, conic, and arrangement2, and serves as a reference implementation for the sweep-line algorithm.

The first phase of the naive algorithm produces a list of all intersections $\left(x, C_{i}, D_{j}\right)$. We generate two conic points $\left(x, C_{i}\right)$ and $\left(x, D_{j}\right)$ for each intersection and sort them using compare_xy. Then it is easy to remove duplicates, and the vertices of the arrangement $A$ are known. We create additional vertices for the points of vertical tangency and also create a dictionary that maps conic points to the vertices of $A$. Then we sort our conic points a second time. For the second sorting, the conic arc is the main key and the second key is the $x$ coordinate. We obtain the sorted list of intersection points on each arc. Using the map from conic points to vertices of $A$, we create the edges of $A$. Finally, we determine the cyclic order of the edges incident to each vertex using the predicate compare_right_of_common_point. The final result is a planar map.

We checked the correctness of the implementation both manually (for small examples) and by checking Euler's equation for planar maps, which is a good heuristic test. We ran the algorithm on: (1) conics in general position, (2) conics with carefully designed degeneracies, and (3) conics with perturbed degeneracies (i.e., almost degenerate points). Figure 2 shows two examples of the second kind. Both feature high-degree intersection points with various multiplicities of intersections. The example on the left also contains intersection points with equal $x$ coordinate. Table 1 shows that, as one would expect, running time increases when degeneracies are present and also when the input precision is increased to represent nearly degenerate cases. However, the increase is not unreasonable. With the naive algorithm, we are able to compute, an arrangement of 200 conics with approximately 55000 intersection points in around 28 minutes. 

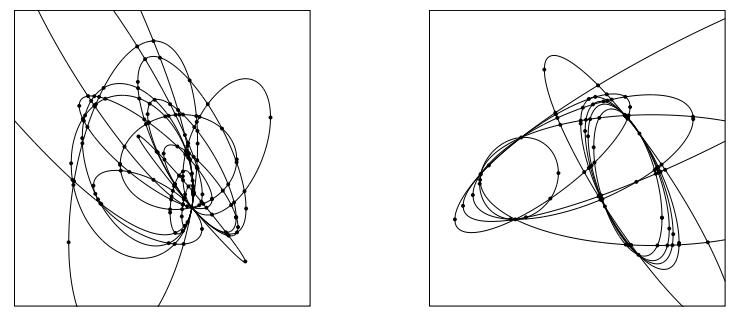

Fig. 2. On the left, a set of 15 ellipses all of which intersect at one point in the lower right quadrant of the picture with varying multiplicities. On the right is a set of 10 ellipses, also in highly degenerate positions

A Generic Implementation of the Sweep Algorithm: Our implementation of the algorithms, classes and predicates described in Sections 3 and 4 is based on the CGAL geometry kernel and the original implementations in LEDA of the sweepline algorithm and the generalized polygons. To easily accommodate different segment types, point types and predicate implementations, we have followed the generic programming paradigm [22] and used the concept of geometric traits classes introduced with CGAL [14]. By supplying different traits classes, the same algorithm can be applied to different kinds of objects or using different predicate implementations.

Such a generic implementation made light work of producing the empirical results presented below that compare different segment types, different predicate implementations and different underlying kernels.

Sweeping Circular Arcs and Straight Line Segments: When only circular arcs and line segments are used, the implementation of the predicates and functions become easier since the coordinates of all intersection points are one-root-numbers. This means in particular that when the circles and lines supporting the segments are specified through rational points, much of the computation can be carried out using rational numbers. Only when it is time to compute the coordinates of the points using the sqrt function will the leda-real number type be used. This is generally a big efficiency win. Furthermore, we are able to exploit the fact that the intersection point between any two rational circles can be described via the

Table 1. Average computation time required by the naive algorithm per pair of conics

\begin{tabular}{l|l|l} 
& $\begin{array}{l}\text { processing time per } \\
\text { pair of conics }\end{array}$ & input precision \\
\hline general position & $30 \mathrm{~ms}$ & $50 \mathrm{bits}$ \\
degenerate position & $48 \mathrm{~ms}$ & $50 \mathrm{bits}$ \\
perturbed degenerate position & $48 \mathrm{~ms}$ & $100 \mathrm{bits}$
\end{tabular}



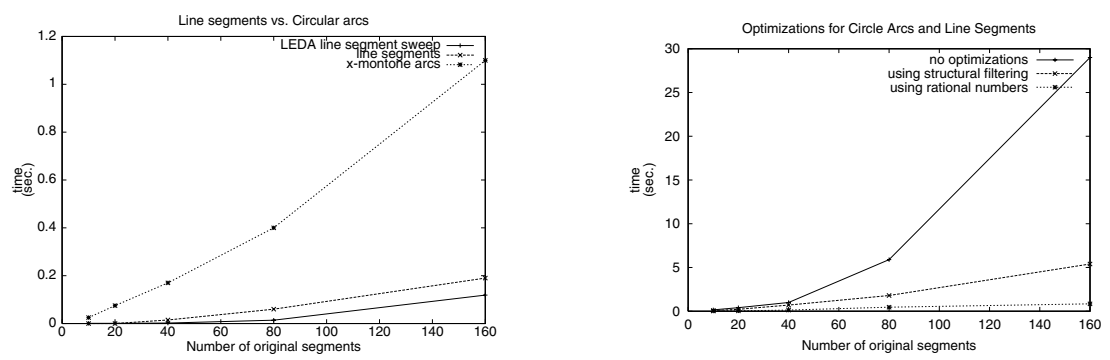

Fig. 3. On the left, a comparison of running times for the optimized version of the LEDA line segment sweep algorithm, our generic implementation of the sweepline algorithm using line segments and the same implementation using circular arcs. On the right, a comparison of running times using various optimizations for circular arcs and line segments. Running times were recorded on an $846 \mathrm{MHz}$ Pentium III processor
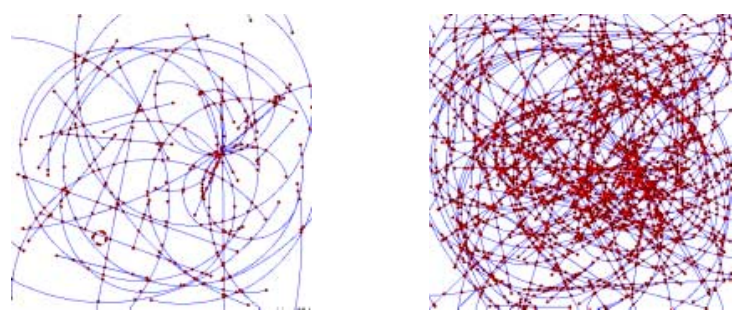

Fig. 4. On the left the planar graph that results from the sweep of a set of 50 circular arcs and line segments with many degeneracies. The graph was produced in less than 1 second on an $846 \mathrm{MHz}$ Pentium III processor. On the right is an example with 241 circular arcs and line segments, which required approximately 2 seconds to compute

intersection of a rational line and a rational circle [10] in our implementation of the compare_xy predicate for points that lie on circular arcs or line segments. That is, before comparing the actual coordinates of the points $p$ and $q$, we first determine if they were constructed in the same way. If so, they are equal and we are done. This technique is called structural filtering [16, 25]. Notice that, in contrast to usual numeric filters used in the exact computation paradigm, which filter out the easy cases that can be dealt with quickly by imprecise number types (i.e., when the points' coordinates are vastly different from each other), this filtering technique works by filtering out the most difficult case (when the points' coordinates are identical) and thus assures that the cases left are usually relatively easy. The graph in figure 3 illustrates the advantages of using these optimizations.

Sweeping Conic Segments: When constructing arrangements of general conic segments such as the ones shown in Figure 2, running times are naturally higher. 
Examples similar to the ones shown in Figure 2 with 30 and 60 conic segments require, respecitvely, 19 seconds and 49 seconds using our current implementation. Table 1 indicates that, as one would expect, running time degrades when degeneracies are introduced. However, with the application of appropriate filtering techniques in our predicate implementations we are confident that the running times for conic segments will come in line with those for circular arcs.

\section{Conclusions}

We described an exact kernel for conic arcs, algorithms for dealing with lowdegree algebraic numbers, a sweep-line algorithm for curved segments, and algorithms for boolean operations on conic polygons. Our algorithms and their implementations are complete, exact, and efficient. We feel that it was crucial for our work that we had all three goals in mind right from the beginning of our work.

\section{References}

[1] J. Bentley and T. Ottmann. Algorithms for reporting and counting geometric intersections. IEEE Transaction on Computers C 28, pages 643-647, 1979. 175, 176

[2] R. Bix. Conics and Cubics: A Concrete Introduction to Algebraic Curves. Springer Verlag, 1998. 177

[3] J.-D. Boissonnat and F. P. Preparata. Robust plane sweep for intersecting segments. Research Report 3270, INRIA, Sophia Antipolis, Sept. 1997. 176

[4] J.-D. Boissonnat and J. Snoeyink. Efficient algorithms for line and curve segment intersection using restricted predicates. In Proc. 15th Annu. ACM Sympos. Comput. Geom., pages 370-379, 1999. 176

[5] C. Burnikel, S. Funke, K. Mehlhorn, S. Schirra, and S. Schmitt. A separation bound for real algebraic expressions. In ESA 2001, volume 2161 of LNCS, pages 254-265, 2001. 180

[6] T. M. Chan. Reporting curve segment intersection using restricted predicates. Computational Geometry, 16(4):245-256, 2000. 176

[7] G. E. Collins and A.-G. Akritas. Polynomial real root isolation using Descartes' rule of sign. In SYMSAC, pages 272-275, Portland, OR, 1976. 179

[8] D. Cox, J. Little, and D. O'Shea. Ideals, Varieties, and Algorithms. SpringerVerlag New York, Inc., 2nd edition, 1997. 179

[9] M. de Berg, M. Kreveld, M. Overmars, and O. Schwarzkopf. Computational Geometry: Algorithms and Applications. Springer, 1997. 177

[10] O. Devillers, A. Fronville, B. Mourrain, and M. Teillaud. Exact predicates for circle arcs arrangements. In Proc. 16th Annu. ACM Sympos. Comput. Geom., 2000. 176, 184

[11] D. P. Dobkin and D. L. Souvaine. Computational geometry in a curved world. Algorithmica, 5:421-457, 1990. 176

[12] D. P. Dobkin, D. L. Souvaine, and C. J. Van Wyk. Decomposition and intersection of simple splinegons. Algorithmica, 3:473-486, 1988. 176 
[13] L. Dupont, D. Lazard, S. Lazard, and S. Petitjean. A new algorithm for the robuts intersection of two general quadrics. submitted to Solid Modelling 2002. 176

[14] A. Fabri, G.-J. Giezeman, L. Kettner, S. Schirra, and S. Schönherr. On the design of CGAL, the computational geometry algorithms library. Software Practice and Experience, 30:1167-1202, 2000. 183

[15] E. Flato, D. Halperin, I. Hanniel, and O. Nechushtan. The design and implementation of planar maps in CGAL. In Proceedings of the 3rd Workshop on Algorithm Engineering, volume 1668 of Lecture Notes in Computer Science, pages 154-168. Springer, 1999. 174

[16] S. Funke and K. Mehlhorn. Look - a lazy object-oriented kernel for geometric computation. In Proceedings of the 16th Annual Symposium on Computational Geometry (SCG-00), pages 156-165, Hong Kong, China, June 2000. Association of Computing Machinery (ACM), ACM Press. 184

[17] N. Geismann, M. Hemmer, and E. Schömer. Computing a 3-dimensional cell in an arrangement of quadrics: Exactly and actually. In ACM Conference on Computational Geometry, 2001. 176

[18] M. Hemmer. Reliable computation of planar and spatial arrangements of quadrics. Master's thesis, Max-Planck-Institut für Informatik, 2002. 179

[19] J. Keyser, T. Culver, M. Foskey, S. Krishnan, and D. Manocha. Esolid - a system for exact boundary evaluation. submitted to Solid Modelling 2002. 176

[20] J. Keyser, T. Culver, D. Manocha, and S. Krishnan. MAPC: A library for efficient and exact manipulation of algebraic points and curves. Technical Report TR98-038, University of N. Carolina, Chapel Hill, 1998. 176

[21] K. Mehlhorn and S. Näher. The LEDA Platform for Combinatorial and Geometric Computing. Cambridge University Press, 1999. 1018 pages. 174, 177, 178,180

[22] D. R. Musser and A. A. Stepanov. Generic programming. In 1st Intl. Joint Conf. of ISSAC-88 and AAEC-6, pages 13-25. Springer LNCS 358, 1989. 183

[23] F. Rouillier and P. Zimmermann. Efficient isolation of polynomial real roots. Technical Report 4113, INRIA, 2001. 179

[24] A. A. Schäffer and C. J. Van Wyk. Convex hulls of piecewise-smooth Jordan curves. J. Algorithms, 8:66-94, 1987. 176

[25] R. Wein. High-level filtering for arrangements of conic arcs. In Proceedings of ESA 2002, 2002. 174, 176, 184 